\title{
The structure and kinematics of the ISM around HD 192281
}

\author{
E. M. Arnal ${ }^{1,2, \star}$, S. Cichowolski ${ }^{1,3, \star \star}$, S. Pineault ${ }^{4}$, J. C. Testori ${ }^{1, \star \star \star}$, and C. E. Cappa ${ }^{1,2, \star}$ \\ ${ }^{1}$ Instituto Argentino de Radioastronomía (CCT-La Plata, CONICET), CC No. 5, 1894 Villa Elisa, Argentina \\ e-mail: arnal@iar.unlp.edu.ar \\ 2 Facultad de Ciencias Astronómicas y Geofísicas, Universidad Nacional de La Plata, Paseo del Bosque s/n, 1900 La Plata, Argentina \\ 3 Instituto de Astronomía y Física del Espacio, CC No. 67, Ciudad Autónoma de Buenos Aires, Argentina \\ ${ }^{4}$ Département de physique, de génie physique et d'optique et Centre de recherche en astrophysique du Québec (CRAQ), \\ Université Laval, Québec (Qué), G1V 0A6, Canada
}

Received 13 October 2010 / Accepted 3 March 2011

\section{ABSTRACT}

\begin{abstract}
Aims. This work aims at investigating the interaction of HD 192281 (O5 Vn((f))p) with its local ISM. The purpose is to analyse the effects that a massive star has on the structure and kinematics of its surrounding gas.

Methods. To carry out this study, we used high-resolution radio continuum and 21-cm $\mathrm{H}$ i line data from the Canadian Galactic Plane Survey (CGPS). This data base was complemented with radio continuum, infrared, and molecular data retrieved from available surveys. Given that HD 192281 is very likely a runaway star, we attempt to establish whether the observed Hi distribution can be interpreted in terms of a simple bow shock model.

Results. Analysis of the $\mathrm{H}_{\mathrm{I}}$ data shows the presence of an $\mathrm{H}_{\mathrm{I}}$ feature likely to be associated with HD 192281. This feature remains detectable from $\sim 5$ to $-10 \mathrm{~km} \mathrm{~s}^{-1}$. At the most negative radial velocities, the shape of the $\mathrm{H}_{\mathrm{I}}$ feature is highly reminiscent of a bow shock structure seen projected onto the plane of the sky. This feature has counterparts at radio-continuum, infrared, and CO emissions. The analysis of the radio continuum data suggests a thermal spectrum for the gas related to this structure.
\end{abstract}

Key words. stars: individual: HD 192281 - stars: mass-loss - stars: winds, outflows - ISM: bubbles - ISM: structure

\section{Introduction}

Over their lifetimes, O-stars have a substantial impact on their local interstellar medium (ISM) through their high Lyman continuum flux, their strong stellar wind, and their eventual disruption as supernova. A subclass of these stars, known as Of stars, contain broad and strong emission lines in their optical spectrum mainly because of the He II ( $\lambda 4686 \AA)$, N III $(\lambda \lambda 4634$, 4640, $4641 \AA)$, and forbidden lines, along with normal absorption lines. These lines frequently show P-Cygni profiles that are indicative of high-mass loss rate. According to Eenens \& Williams (1994) and Chlebowski \& Garmany (1991), the mass loss rate $(\dot{M})$ and wind terminal velocity $\left(v_{\infty}\right)$ of Of stars range from $10^{-7}$ to $10^{-5} M_{\odot} \mathrm{yr}^{-1}$ and from 2000 to $3000 \mathrm{~km} \mathrm{~s}^{-1}$, respectively. A total of 108 Of stars are known to exist in the Galaxy, and almost half of them are associated with optical nebulae (Lozinskaya 1992).

Since massive stars having a main sequence mass above $10 M_{\odot}$ radiate a copious rain of ionizing photons into their local ISM $\left(1.8 \times 10^{48} \leq N_{\text {Lyc }} \leq 7.1 \times 10^{49} \mathrm{~s}^{-1}\right.$, Martins et al. 2005), and inject into it a great deal of mechanical energy via their powerful stellar winds $\left(L_{w}=0.5 \times \dot{M} \times v_{\infty}^{2}\right)$, they have the basic ingredients for profoundly affecting the structure and dynamics of the ISM. Based on the early works of Avedisova (1972) and Weaver et al. (1977), it was soon realized that massive stars can create what is known as an interstellar bubble (IB)in their

\footnotetext{
* Member of Carrera del Investigador Científico, CONICET-CCT La Plata, Argentina.

$\star \star$ Member of Carrera del Investigador Científico, CONICET, Argentina.

$\star \star \star$ Member of Carrera Personal de Apoyo, CONICET-CCT La Plata, Argentina.
}

surrounding ISM. This is a region of very low density $\left(n \sim 10^{-2}\right.$ to $\left.10^{-3} \mathrm{~cm}^{-3}\right)$ and high temperature $\left(T \sim 10^{6-7} \mathrm{~K}\right)$, which is surrounded by an expanding shell of gas that may be detectable as an expanding $\mathrm{H}_{\mathrm{I}}$ shell after cooling down. In the ideal case where the star is at rest with respect to its surroundings and the ISM is homogeneous, theoretical models predict a spherically symmetric IB with the massive star located at its centre. On the other hand, in those cases where the stellar peculiar velocity is high enough $\left(v_{\text {pec }} \geq 30 \mathrm{~km} \mathrm{~s}^{-1}\right)$, the spherically symmetric geometry will be distorted, and a bow-shock geometry, with the high-velocity star located close to its apex, will be observed. Observationally, most of the high-mass stars have low peculiar spatial velocities, and when observed in the $\lambda \sim 21-\mathrm{cm}$ line radiation, the star is usually seen projected onto, or close to, a minimum in the $\mathrm{H}_{\mathrm{I}}$ emissivity. Furthermore, since dust particles are mixed with gaseous matter they may emit in the far infrared, after being heated up by the stellar radiation. Therefore, MSX and IRAS databases are also well suited to these studies.

Most of the earlier H I studies toward O-stars (see Cappa $\&$ Herbstmeier 2000, and references there in) were carried out using single-dish instruments having an angular resolution in the range from $\sim 9^{\prime}$ to $30^{\prime}$. These studies show that most of the stars appear projected towards regions of the sky having a low neutral hydrogen emissivity when compared to their surroundings. The shape of these low emission regions varies from almost spherical to highly elliptical, and can only be observed in a limited range of radial velocity.

The situation for radio observations, reviewed by Cappa et al. (2003), has now evolved considerably with the advent of large galactic surveys, namely the Canadian Galactic Plane Survey (CGPS, Taylor et al. 2003), the Southern GPS (SGPS, McClureGriffiths et al. 2005; Haverkorn et al. 2006), and the VLA GPS 
(VGPS, Stil et al. 2006). These surveys now make it possible to identify and study the structure and dynamics of the ISM at $\sim 1$ arcmin resolution.

We have undertaken a project aimed at systematically studying the immediate vicinity of a number of stars expected to be major contributors in shaping the ISM (Cappa et al. 2002; Cichowolski et al. 2003; Pineault et al. 2008). To minimize confusion, we selected objects with relatively high galactic latitudes. In this paper, we analyse the environs of the Of star HD 192281. This star, which is a member of the OB-association Cyg OB8, is located at $(l, b)=\left(77^{\circ} .12,+3^{\circ} .40\right)$, has spectral type $05 \mathrm{Vn}((\mathrm{f})) \mathrm{p}$ (Walborn 1972), and is a fast rotator with $V \sin i=270 \mathrm{~km} \mathrm{~s}^{-1}$ (Conti \& Ebbets 1977). Distance determinations for this star vary between $1.55 \mathrm{kpc}$ (Stone 1978) and $2.4 \mathrm{kpc}$ (Humphreys 1978).

The kinematics of this star is a matter of some debate. On one hand, based on a comparison between the observed radial velocity, $V_{\mathrm{r}} \simeq-48 \mathrm{~km} \mathrm{~s}^{-1}$ and the radial component of the circular velocity that would correspond to the actual location of the star within the Galaxy, Cruz-González et al. (1974) derived a peculiar radial velocity of $-55 \mathrm{~km} \mathrm{~s}^{-1}$ and list this star as a "probable runaway". On the other hand, Gies \& Bolton (1986) (GB) cast some doubts on this interpretation based on evidence of a Balmer line velocity progression in the optical spectrum of HD192281. They claim that the radial velocity derived from the He II line $\lambda 4686,-27.1 \mathrm{~km} \mathrm{~s}^{-1}$, is probably closer to the physical velocity of the star, because this line is formed deeper in the stellar atmosphere, where the wind velocity is lower. Based on this, GB concluded that HD 192281 is unlikely to be a runaway star. Barannikov (1993) performed a radial velocity variability analysis of this star, and claim to find radial velocity variations with a semi-amplitude of $16.1 \pm 2.4 \mathrm{~km} \mathrm{~s}^{-1}$ on a 5.48 day period. $\mathrm{He}$ further argues that this small amplitude is compatible with the presence of a low-mass companion star and derived a system's baricentric radial velocity of $-40.4 \pm 2.7 \mathrm{~km} \mathrm{~s}^{-1}$. These results should be viewed with caution, since Barannikov's claims rely on measurements of the strongest absorption lines of the Balmer series ( $\mathrm{H} 9, \mathrm{H} 8, \mathrm{H} \delta, \mathrm{H} \gamma$, and $\mathrm{H} \beta$ ) and $\mathrm{He} \mathrm{I} \lambda 4026$ and $\mathrm{He}$ II $\lambda \lambda$ 4200, 4562 and 4686 lines. De Becker \& Rauw (2004) carried out a detailed radial velocity analysis of the He II $\lambda 4541$ absorption line, which forms deeper inside the stellar photosphere, and derived a mean radial velocity of $-23.1 \pm 8 \mathrm{~km} \mathrm{~s}^{-1}$. They find no evidence in favour of a radial velocity variability as claimed by Barannikov. In this paper all radial velocities are referred to the local standard of rest.

Proper motion measurements for HD 192281 are available from several catalogues. Hipparcos (ESA 1997) quotes $\left(\mu_{\alpha} \cos \delta\right.$, $\left.\mu_{\delta}\right)=(-7.76 \pm 0.53,-2.54 \pm 0.56) \mathrm{mas} \mathrm{yr}^{-1}$, the Tycho-2 Catalogue (Hog et al. 2000) lists $\left(\mu_{\alpha} \cos \delta, \mu_{\delta}\right)=(-7.9 \pm 1.0$, $-1.8 \pm 1.1)$ mas $^{-1}{ }^{-1}$, while the Third US Naval Observatory CCD Astrograph Catalog (UCAC3) gives $\left(\mu_{\alpha} \cos \delta, \mu_{\delta}\right)=(-8.0 \pm$

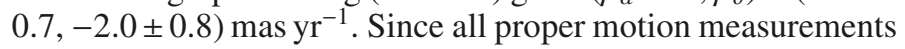
are, within errors, compatible with each other, in this paper we shall adopt those given by Hipparcos. Moffat et al. (1998) claimed that this star has significant peculiar proper motions, supporting a possible runaway nature for this star.

\section{Observational data}

This research was carried out using a number of surveys and databases (see Table 1) publicly available, namely:

a) The CGPS. It is a high-resolution ( $\sim 1$ arcmin $) \mathrm{H}_{\mathrm{I}}$ line and 408 and $1420 \mathrm{MHz}$ radio continuum survey of the Galactic
Table 1. Main parameters of the datasets.

\begin{tabular}{|c|c|}
\hline \multirow{2}{*}{\multicolumn{2}{|c|}{$\begin{array}{l}\text { Line Databases } \\
\mathrm{H}_{\text {I }} \text { line }\end{array}$}} \\
\hline & \\
\hline Synthesized beam & $1.5 \times 1{ }^{\prime} .0$ \\
\hline Position angle & $55: 8$ \\
\hline Brightness temperature rms noise $(\mathrm{K})$ & 2.1 \\
\hline Channel separation $\left(\mathrm{km} \mathrm{s}^{-1}\right)$ & 0.83 \\
\hline Velocity resolution $\left(\mathrm{km} \mathrm{s}^{-1}\right)$ & 1.3 \\
\hline Central velocity $\left(\mathrm{km} \mathrm{s}^{-1}\right)$ & -60.0 \\
\hline Velocity coverage $\left(\mathrm{km} \mathrm{s}^{-1}\right)$ & 211 \\
\hline \multicolumn{2}{|l|}{$\mathrm{CO}$ line } \\
\hline Angular resolution & $8: 7$ \\
\hline rms noise $(\mathrm{K})$ & 0.07 \\
\hline Velocity resolution $\left(\mathrm{km} \mathrm{s}^{-1}\right)$ & 1.3 \\
\hline Central velocity $\left(\mathrm{km} \mathrm{s}^{-1}\right)$ & -30.55 \\
\hline Velocity coverage $\left(\mathrm{km} \mathrm{s}^{-1}\right)$ & 219.7 \\
\hline \multicolumn{2}{|l|}{ Radio continuum databases } \\
\hline \multicolumn{2}{|l|}{$408 \mathrm{MHz}$} \\
\hline Synthesized beam & $4.5 \times 2.8$ \\
\hline Position angle & 57.1 \\
\hline Measured rms noise $(\mathrm{K})$ & $\sim 1.1$ \\
\hline \multicolumn{2}{|l|}{$1420 \mathrm{MHz}$} \\
\hline Synthesized beam & $1.3 \times 0.9$ \\
\hline Position angle & 57.5 \\
\hline Measured rms noise $(\mathrm{K})$ & $\sim 0.06$ \\
\hline \multicolumn{2}{|l|}{$2695 \mathrm{MHz}$} \\
\hline Angular resolution & 4.3 \\
\hline Measured rms noise (Jy) & 0.67 \\
\hline \multicolumn{2}{|l|}{$8350 \mathrm{MHz}$} \\
\hline Angular resolution & $9: 7$ \\
\hline Measured rms noise (Jy) & 0.16 \\
\hline \multicolumn{2}{|l|}{$14350 \mathrm{MHz}$} \\
\hline Angular resolution & 6.6 \\
\hline Measured rms noise (Jy) & 1.78 \\
\hline \multicolumn{2}{|l|}{ Infrared databases } \\
\hline \multicolumn{2}{|l|}{ MSX } \\
\hline Angular resolution & $18^{\prime \prime}$ \\
\hline Measured rms noise (Band A)(Jy/arcmin $\left.{ }^{2}\right)$ & 7.6 \\
\hline Measured rms noise $\left(\right.$ Band C) $\left(\mathrm{Jy} / \operatorname{arcmin}^{2}\right)$ & 17.0 \\
\hline Measured rms noise (Band D)(Jy/arcmin $\left.{ }^{2}\right)$ & 51.0 \\
\hline Measured rms noise (Band E)(Jy/arcmin²) & 108.0 \\
\hline \multicolumn{2}{|l|}{ IRAS - HIRES } \\
\hline Angular resolution $(60 \mu \mathrm{m})$ & $1.85 \times 1.02$ \\
\hline Beam Position angle $(60 \mu \mathrm{m})$ & $217^{\circ}$ \\
\hline Measured rms noise $(60 \mu \mathrm{m})\left(\mathrm{Jy} / \operatorname{arcmin}^{2}\right)$ & 54.0 \\
\hline Angular resolution $(100 \mu \mathrm{m})$ & $2 ! 33 \times 1 ! 92$ \\
\hline Beam position angle $(100 \mu \mathrm{m})$ & $217^{\circ}$ \\
\hline Measured rms noise $(100 \mu \mathrm{m})\left(\mathrm{Jy} / \mathrm{arcmin}^{2}\right)$ & 690.0 \\
\hline
\end{tabular}

plane obtained with the Synthesis Telescope (Landecker et al. 2000) of the Dominion Radio Astrophysical Observatory (DRAO). It provides a unique opportunity to carry out an unbiased homogeneous study of the ISM structures and dynamics associated with massive stars and their evolved descendants. Before assembly into a mosaic, the data for the individual fields are carefully processed to remove artifacts and to obtain the highest possible dynamic range, using the routines described by Willis (1999). Accurate representation of all structures on the largest scales is assured by incorporating data from large single antennas with data from the Synthesis Telescope, after suitable filtering in the Fourier domain. Continuum data are derived from the $1.4 \mathrm{GHz}$ Effelsberg survey (Reich et al. 1997) and the $408 \mathrm{MHz}$ All-Sky Survey by Haslam (1985). Single antenna H I data are obtained from a survey of the CGPS region made with the DRAO 26-m Telescope (Higgs \& Tapping 2000). 


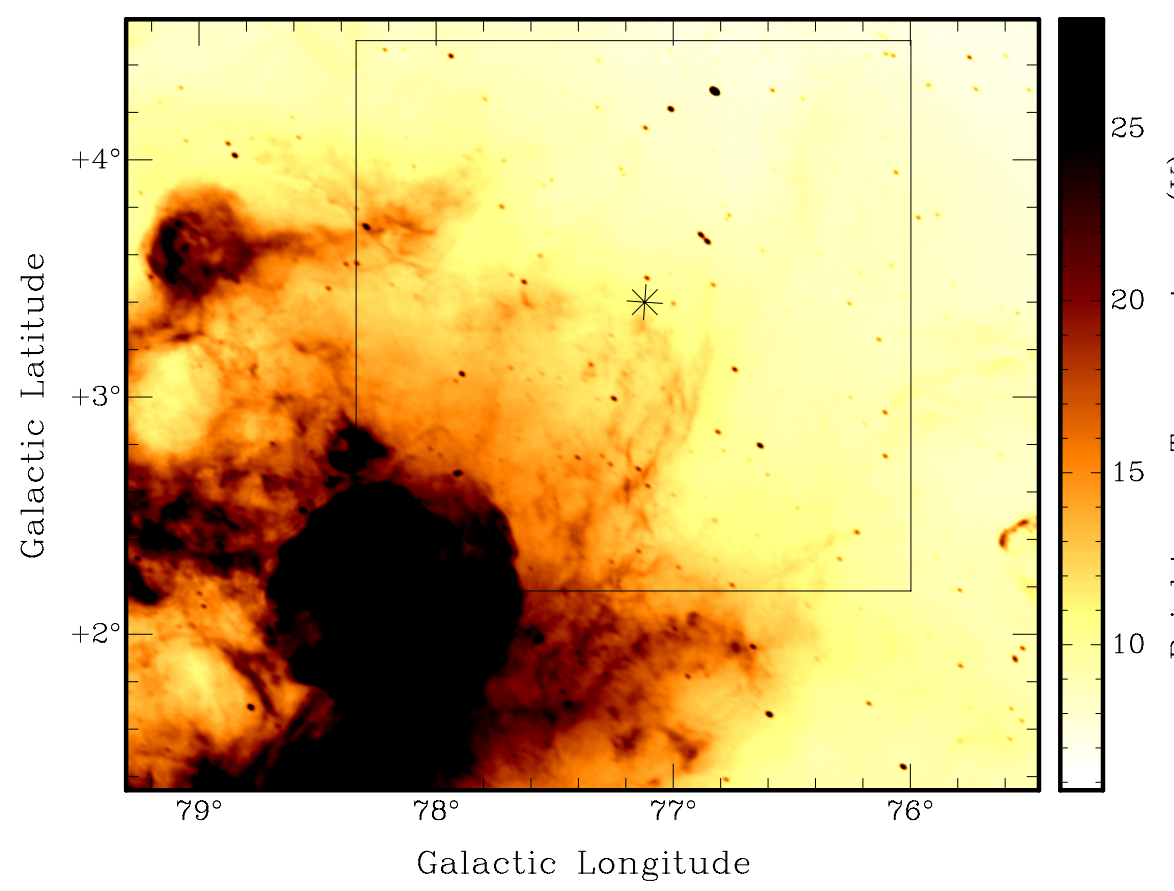

Fig. 1. Large-scale CGPS $1420 \mathrm{MHz}$ continuum emission towards HD 192281 and its surroundings. The stellar position is indicated by an asterisk. The square shows the region covered by the other figures of this paper. The strong continuum source located in the lower left corner of this figure corresponds to the Galactic supernova remnant G78.2+2.1.
The CGPS database also comprises IRAS high-resolution (HIRES) images (Fowler \& Aumann 1994; Cao et al. 1997) produced at the Infrared Processing and Analysis Center (IPAC) ${ }^{1}$. The image is the result of 20 iterations of the algorithm, giving an approximate resolution of $1^{\prime}-2^{\prime}$.

b) Additional radio continuum surveys carried out at $2695 \mathrm{MHz}$ (Fürst et al. 1990), $8350 \mathrm{MHz}$, and $14350 \mathrm{MHz}$ (Langston et al. 2000).

c) Infrared data retrieved from the Midcourse Space Experiment $\left(\mathrm{MSX}^{2}\right)$ (Price et al. 2001).

d) Carbon monoxide data $(J=1 \rightarrow 0)$ retrieved from the survey carried out by Dame et al. (1987).

\section{Results}

\subsection{Radio continuum emission}

Figure 1 is a $1420 \mathrm{MHz}$ CGPS continuum image showing the large-scale structure of the ISM in the general direction to HD $192281\left[(l, b)=\left(77^{\circ} .12,+3.40\right)\right]$. The strong and almost circular feature seen in the lower left part of the image is the well-known shell-type supernova remnant G78.2+2.1 (EDR4, $\gamma$ Cygni), recently thoroughly studied by Ladouceur \& Pineault (2008). A large number of point sources, most of them extragalactic in nature, are evident at high galactic latitudes. The high dynamic range of the CGPS data allows the detection of weak emission features present in the immediate surroundings of G78.2+2.1. In particular, between G78.2+2.1 and the position of HD 192281 there is evidence of two weak continuum features. Figure 2 shows that these features are detectable at $408 \mathrm{MHz}$, and become better defined at $2695 \mathrm{MHz}$. At this frequency the continuum emission in the neighborhood of HD 192281 has the appearance of an incomplete shell-like feature with the star close

\footnotetext{
1 IPAC is funded by NASA as part of the Infrared Astronomical Satellite (IRAS) extended mission under contract to the Jet Propulsion Laboratory (JPL).

2 IPAC provides access to the MSX and IRAS images through the Infrared Science Archive at http://irsa.ipac.caltech.edu
}

to its highest latitude extreme. Ladouceur \& Pineault (2008) suggested that this faint shell could have resulted from a breakout of the $S N R$ blast wave into a medium of lower density.

\subsection{Extended infrared emission}

The large shell-like structure observed in the radio continuum emission distribution is also easily recognizable at infrared wavelengths. The MSX at $8.3 \mu \mathrm{m}$ along with the 12,60 , and $100 \mu \mathrm{m}$ HIRES images, are shown in Fig. 3. At IR wavelengths, an incomplete ring-like structure similar to that observed at $2695 \mathrm{MHz}$ is observed.

In the immediate vicinity of HD 192281 the IR emission shows a small ring-like feature of about $17^{\prime}$ in size centred near the stellar position. This small structure is clearly identified in MSX at $8.3 \mu \mathrm{m}$ emission (see the upper left image of Fig. 3) and in the four IRAS bands. The star lies near the border of the cavity in all IRAS bands. Though at first glance one may be tempted to physically relate the IR feature to the star, it is worth stressing that the large-scale IR emission is highly filamentary, and that several small IR structures similar to the one just described are easily recognizable towards different regions (see for example those features centred at $(l, b)=\left(77^{\circ} .01,2^{\circ} .93\right.$ ) and $(l, b)=\left(77^{\circ} .65,2.67\right)$ of the large-scale IR shell-like feature. Therefore, the presence of a small ring of IR emission seen projected onto HD 192281 may well be a chance coincidence between the star and one of the many small scale IR features that constitute the extended IR emission.

\subsection{Neutral hydrogen line emission}

The neutral hydrogen emission spans the velocity range from -130 to $+30 \mathrm{~km} \mathrm{~s}^{-1}$ along $l \simeq 77^{\circ}$. To increase the signal-tonoise $(\mathrm{S} / \mathrm{N})$ ratio, the original line channel maps were spatially convolved to yield a Gaussian beam response of $F W H M=3^{\prime}$. In this way, the final rms noise level, in units of brightness temperature, in a single line channel map is $\Delta T_{\mathrm{rms}} \sim 0.4 \mathrm{~K}$. To help us distinguish between $\mathrm{H}_{\mathrm{I}}$ minima possibly associated with 


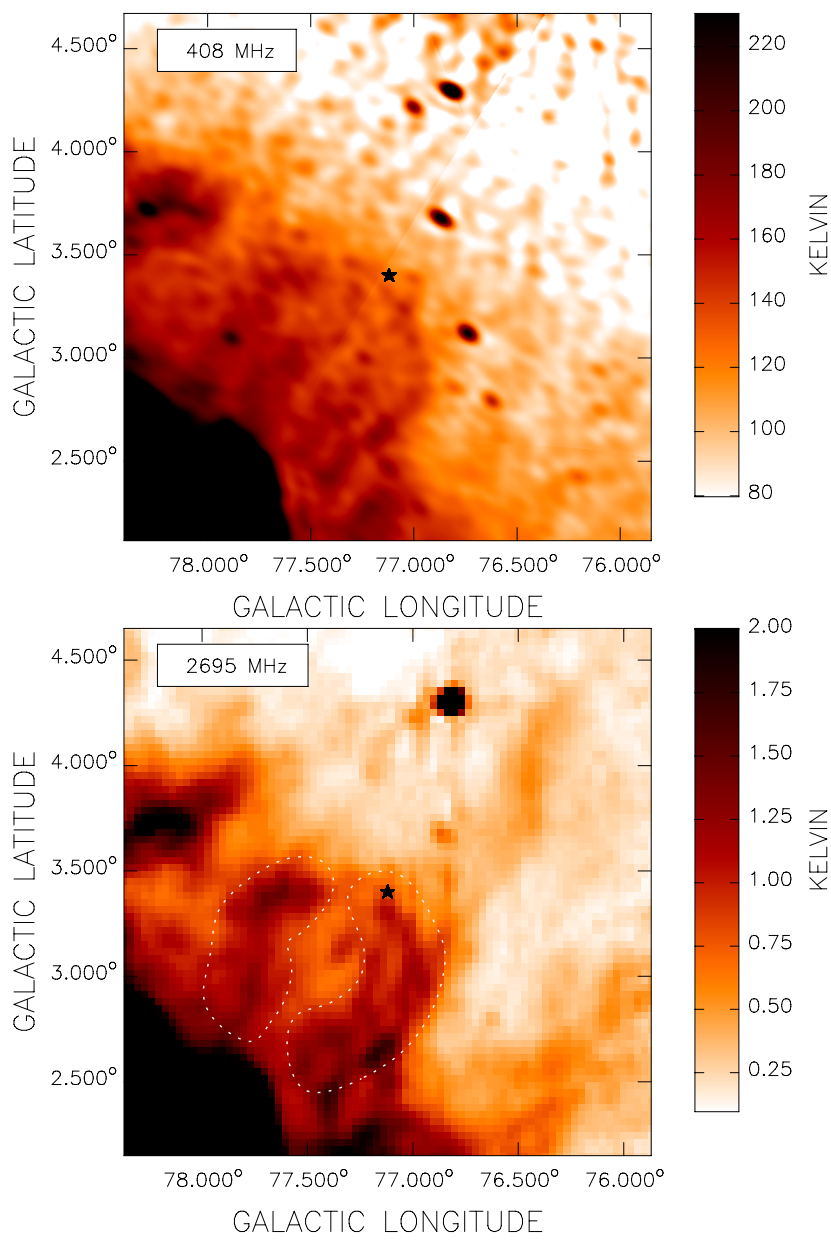

Fig. 2. Radio continuum emission towards HD 192281. The upper and lower panels show the emission at 408 and $2695 \mathrm{MHz}$, respectively. The position of HD 192281 is marked by a star symbol. The white dashed contours shown in the lower panel mark the location of those areas used to measure the continuum flux density.

HD 192281 from those arising from the normal structure of the ISM, the following criteria were adopted.

i) The $\mathrm{O}$ star should appear projected onto, or close to, a region of depressed $\mathrm{H}_{\mathrm{I}}$ emissivity.

ii) The region mentioned in i) should remain observable throughout a significant number of individual and consecutive line channel maps. In our case we have set this number to five.

iii) The kinematical distance derived, using existing rotation curves of the Milky Way (i.e. Fich et al. 1989), from the mean radial velocity of the $\mathrm{H}_{\mathrm{I}}$ minimum should not be in conflict with the accepted distance range for the star.

Given that along the galactic longitude $l \sim 77^{\circ}$ the velocity gradient is small, about $6.5 \mathrm{~km} \mathrm{~s}^{-1} \mathrm{kpc}^{-1}$, making the task of determining a "reliable" kinematic distance particularly difficult, in the case of HD 192281 the last criterion has a low weight. In addition one should bear in mind that significant non-circular motions are known to exist in this part of the Galaxy.

Considering the criteria just mentioned, the entire $(l, b, v)$ H I data cube was inspected for features likely to be related to HD 192281. In Fig. 4 three averaged line channel maps spanning the velocity range from +15.0 to $-30.3 \mathrm{~km} \mathrm{~s}^{-1}$ are shown.
To facilitate the recognition of features that may arise from the action of the O-star onto its local ISM, a constant mean brightness temperature value was subtracted from every channel map. This mean level is obtained by averaging the brightness temperature over the full field of view of each line channel map. Every image is an average of eighteen individual line channel maps, has an rms noise of about $\Delta T_{\text {rms }} \sim 0.1 \mathrm{~K}$, and covers a velocity interval of about $15 \mathrm{~km} \mathrm{~s}^{-1}$. In those images shown in Figs. $4 \mathrm{a}$ and $\mathrm{c}$, the $\mathrm{H}_{\mathrm{I}}$ emission distribution displays a lot of small angular scale structures superimposed on a brightness temperature gradient characteristic of the overall galactic $\mathrm{H}_{\mathrm{I}}$ emission in this part of the Milky Way. On the other hand, within the velocity interval +0.2 to $-14.65 \mathrm{~km} \mathrm{~s}^{-1}$ (Fig. 4b), the H I distribution drastically departs from the one described above. The dominant feature is now a hollow structure of $\mathrm{H}_{\mathrm{I}}$ emission protruding from an otherwise normal galactic emission. HD 192281 is observed projected onto the high-latitude border of this structure. It is worth mentioning that, although the entire velocity range spanned by the galactic $\mathrm{H}_{\mathrm{I}}$ emission was searched for $\mathrm{H}_{\mathrm{I}}$ features likely to be related to HD 192281, no peculiar structure besides the one shown in Fig. 4b was found.

To better define the spatial structure and velocity behaviour of the $\mathrm{H}_{\mathrm{I}}$ gas in this velocity range, a mosaic of twelve averaged line channel maps spanning the velocity range from $+5.13 \mathrm{~km} \mathrm{~s}^{-1}$ to $-22.08 \mathrm{~km} \mathrm{~s}^{-1}$ are depicted in Fig. 5. Each image covers a velocity range of $\sim 2.5 \mathrm{~km} \mathrm{~s}^{-1}$ and has an rms noise of $\Delta T_{\mathrm{rms}} \sim 0.3 \mathrm{~K}$.

The image of the $\mathrm{HI}_{\mathrm{I}}$ distribution at $+5.13 \mathrm{~km} \mathrm{~s}^{-1}$ shows HD 192281 projected onto a large region of low emissivity. From a morphological point of view, HD 192281 appears close to the northermost tip of a curved $\mathrm{H}_{\mathrm{I}}$ feature that can be traced from $(l, b)=\left(77^{\circ} .25,+2.50\right)$ up to $(l, b)=\left(77^{\circ} .07,+3^{\circ} .30\right)$. A second arc-like feature begins to be noticeable at $0.19 \mathrm{~km} \mathrm{~s}^{-1}$ at $(l, b) \simeq\left(77^{\circ} .23,+3.2\right)$. At more negative velocities, from -7.23 to $-14.66 \mathrm{~km} \mathrm{~s}^{-1}$, this feature becomes more prominent and beyond $-17.13 \mathrm{~km} \mathrm{~s}^{-1}$ it loses its identity and fades away.

\subsection{Carbon monoxide emission}

To investigate the presence of $\mathrm{CO}$ gas associated with HD 192281, the distribution of the carbon monoxide (CO ) $J=$ $1 \rightarrow 0$ emission was analysed in the velocity range from -140 to $+80 \mathrm{~km} \mathrm{~s}^{-1}$. In the velocity range from $-11.1 \mathrm{~km} \mathrm{~s}^{-1}$ to -16.3 , the bulk of the molecular emission arises from an elongated feature (Fig. 6, lower panel) that reaches its maximum temperature at $(l, b)=\left(77^{\circ} .1,+3^{\circ} .6\right)$. This feature is labelled $\mathrm{B}$. The major axis of this concentration runs almost parallel to the galactic plane. HD 192281 is seen projected towards a small indentation of the outer border of the molecular structure facing the Galactic plane. A second and weaker CO feature (Feature A) is observed in the same figure at $(l, b)=\left(77^{\circ} .6,+3.4\right)$. This feature has a counterpart at continuum and infrared wavelengths. The velocity range where this structure is best detected ranges from -15.0 to $-17.6 \mathrm{~km} \mathrm{~s}^{-1}$.

Within the velocity range from +5.9 to $+0.7 \mathrm{~km} \mathrm{~s}^{-1}$ (see Fig. 6, upper panel), the $\mathrm{CO}$ distribution is quite different from the previous one. Most of the emission comes from a broad and slightly curved structure (Features D and E) that extends from $2.0 \leq b \leq 3.5$ and $76^{\circ} .6 \leq l \leq 76^{\circ} .8$. The latter and the $\mathrm{CO}$ feature peaking at $(l, b)=(77.7,+2.5)$ (Feature C) have a spatial distribution that is highly reminiscent of the incomplete shell-like features observed at IR and radio continuum wavelengths. 

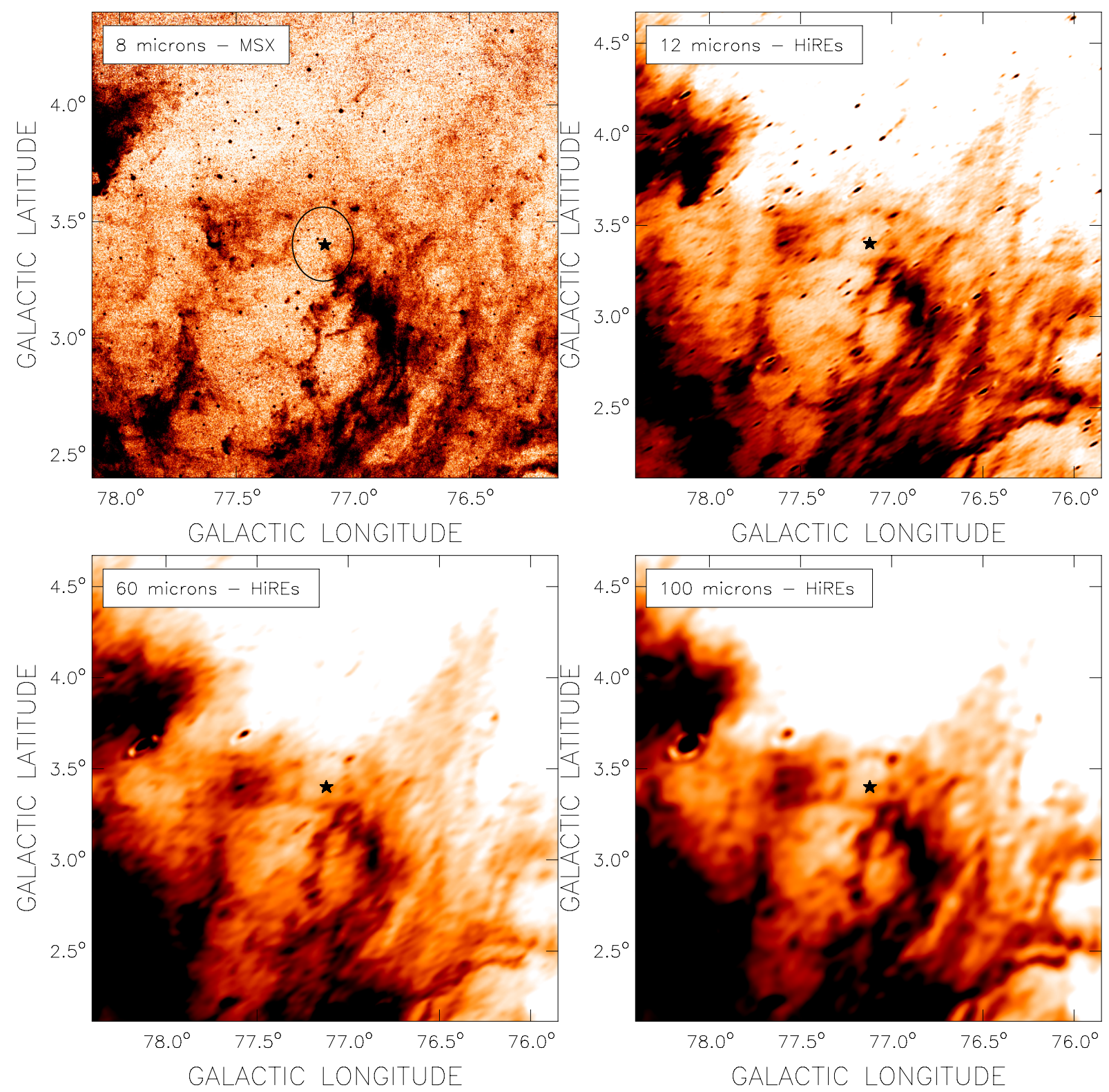

Fig. 3. Extended IR emission towards HD 192281 at $8.3 \mu \mathrm{m}$ (upper left panel), $12.12 \mu \mathrm{m}$ (upper right panel), $60 \mu \mathrm{m}$ (lower left panel), and $100 \mu \mathrm{m}$ (lower right panel). The position of HD 192281 is depicted by a star symbol. In the upper left image, the circumference centred at the stellar location marks the extension of the small ring-like structure referred to in Sect. 3.2.

\section{Discussion}

\subsection{Nature of the radio continuum emission}

To help shed some light on the nature of the shell-like structure observed in the radio continuum emission, its flux density was measured at different frequencies. To estimate the flux density of this source, a background contribution from unrelated gas has to be accounted for. To this end a twisted plane was fitted at every frequency through regions located close enough to, but outside of, the shell-like feature (see Fig. 2). Admittedly the background removal has two main drawbacks that relate to: $i$ ) the extended nature of the source, and $i$ ) the angular proximity to the SNR $\mathrm{G} 78.2+2.1$. Indeed the continuum source we are interested in is seen projected onto a region with a steep brightness temperature gradient that strongly depends on position.
The measured continuum fluxes are given in Table 2. The large errors mainly reflect the uncertainties due to the background removal procedure. The resulting radio spectrum is given in Fig. 7. The best least square fit to these values (Fig. 7) provides a spectral index of $\alpha=-0.11 \pm 0.02$, a value fully compatible with optically thin, thermal, free-free emission. This spectral index rules out the suggestion made by Ladouceur \& Pineault (2008) that this shell may be a breakout of the SNR G78.2+2.1 into a medium of lower density.

\subsection{Physical parameters of the $\mathrm{H} \|$ region}

To infer the properties of the radio continuum emitting region, the formulae from Mezger \& Henderson (1967) for a pure hydrogen plasma were used. The estimate of the physical parameters relies upon assumptions concerning the geometry of the 

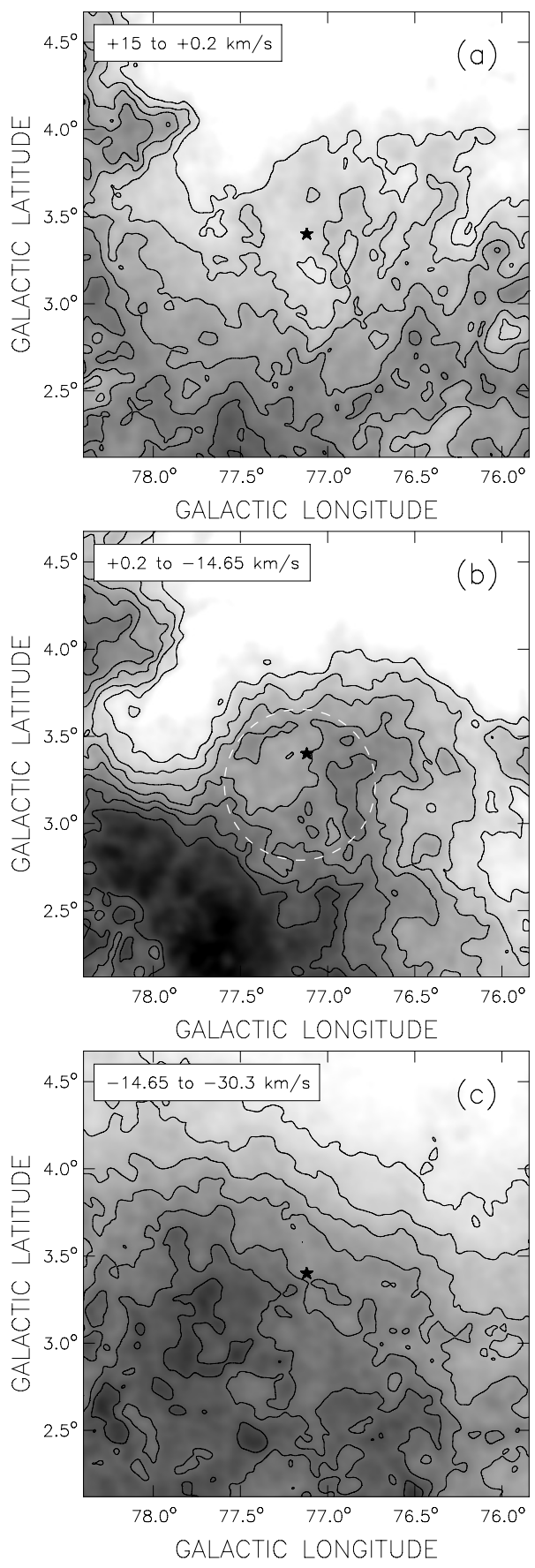

Fig. 4. H I brightness temperature images for selected velocity ranges. The velocity range covered by each map is given in its upper left corner and the displayed temperatures are a mean over the entire velocity width. The lowest temperature contour is $-15 \mathrm{~K}$ and the contour spacing is $5 \mathrm{~K}$. A constant mean brightness temperature value was subtracted from each image (see text). The stellar location is indicated by a star symbol. The white dashed-line circumference drawn in the middle panel marks the position of the Hi hollow feature described in Sect. 3.3.

source. In this case, we assume that the ionized gas is distributed in an incomplete sphere, which lacks a spherical polar cap whose opening angle, as measured from the centre of the ionized gas distribution is $48^{\circ}$. As can be observed from Fig. 2, the interior of the assumed spherical distribution has lower continuum emission, indicating that the sphere is hollow. Thus, the geometry of the source can be best defined by assuming that the ionized gas is present in two concentric spherical shells whose thickness varies
Table 2. Measured continuum flux densities.

\begin{tabular}{lcc}
\hline \hline $\begin{array}{l}\text { Frequency } \\
(\mathrm{GHz})\end{array}$ & $\begin{array}{c}\text { Flux density } \\
(\mathrm{Jy})\end{array}$ & Database \\
\hline 0.408 & $15.9 \pm 1.0$ & CGPS data \\
1.420 & $13.8 \pm 1.2$ & CGPS data \\
2.695 & $13.4 \pm 0.8$ & Fürst et al. (1990) survey \\
8.350 & $11.1 \pm 1.3$ & Langston et al. (2000) survey \\
14.350 & $9.9 \pm 1.8$ & Langston et al. (2000) survey \\
\hline
\end{tabular}

between 0.47 and 0.67 of their outer radius. Taking the assumed geometry into account, we use the spherical model proposed by Mezger \& Henderson (1967) considering a filling factor of about $f=0.8$.

After adopting the galactic disk temperature gradient found by Quireza et al. (2006), an electron temperature of $8160 \pm 530 \mathrm{~K}$ is found. The electron density $\left(n_{\mathrm{e}}\right)$, and the ionized mass $\left(M_{\mathrm{HII}}\right)$ can be obtained using simple geometric models of HII regions (Schraml \& Mezger 1969). Using their spherical model and the volume filling factor quoted above, we derived an electron density of $n_{\mathrm{e}}=7.3 \pm 0.8 \mathrm{~cm}^{-3}$ and a total mass of ionized gas of $M_{\mathrm{HII}}=1300 \pm 650 M_{\odot}$. The total number of Lyman continuum photons needed to keep the region ionized is given by $N_{v}=0.76 \times 10^{47} T_{4}^{-0.45} v^{0.1} S_{v} d^{2}$ (Chaisson 1976), where $T_{\mathrm{e}}$ is the electron temperature in units of $10^{4} \mathrm{~K}, d$ the distance in $\mathrm{kpc}$, $v$ the frequency in units of $\mathrm{GHz}$, and $\mathrm{S}_{v}$ the measured total flux density in units of Jy. We obtained $N_{v}=(3.6 \pm 1.5) \times 10^{48} \mathrm{~s}^{-1}$. The uncertainties quoted for $n_{\mathrm{e}}, M_{\mathrm{HII}}$, and $N_{v}$ are derived under the assumption of a distance error of about $0.3 \mathrm{kpc}$, an electron temperature uncertainty of $530 \mathrm{~K}$ and the flux density error quoted in Table 2. The number of ionizing photons derived in this way is a lower limit to the total number of Lyman continuum photons emitted by the star, since a fraction of them may be absorbed by dust present inside the HII region. After being heated, the dust grains emit at infrared wavelengths. The number of Lyman continuum photons needed to power the $\mathrm{H}_{\text {II }}$ region, could well be provided by HD 192281, since this number is a small fraction of the total UV ionizing photons emitted by an O5 V star $\left(\sim 1.8 \times 10^{49} \mathrm{~s}^{-1}\right)$ like HD 192281 (Martins et al. 2005).

\subsection{Dust parameters}

The large-scale IR emission distribution at four different wavelengths is shown in Fig. 3. At all frequencies, emission arising from an incomplete shell-like feature is observed. This IR feature is highly reminiscent of the one observed at radio continuum frequencies. At IR wavelengths HD 192281 is also observed towards a region of low IR emissivity that is close to the northern extreme of the shell-like distribution. After removing the background emission, the infrared flux densities were determined for all MSX and HIRES bands. The fluxes were derived by integrating over different polygons surrounding the source. The measured flux densities are given in Table 3.

It has been empirically found that the submillimetre spectrum of a cool dust cloud can be fitted by the product of the black-body intensity, $B_{v}\left(T_{\mathrm{d}}\right)$, and a grain emissivity that varies as $v^{m}$, where $m$ increases from $\sim 1$ at $\lambda \leq 200 \mu \mathrm{m}$ to $\geq 2$ at longer wavelengths $(\lambda \sim 1000 \mu \mathrm{m})$ (Schwartz 1982). The dust temperature derived from the fit to the measured 60 and $100 \mu \mathrm{m}$ data of a function of the form $v^{m} \times B_{v}\left(T_{\mathrm{d}}\right)$, adopting $m=1.5$, is of the order of $T_{\mathrm{d}} \simeq 24 \pm 4 \mathrm{~K}$. The error quoted for $T_{\mathrm{d}}$ stems from the uncertainty in the definition of the background zero level, and 

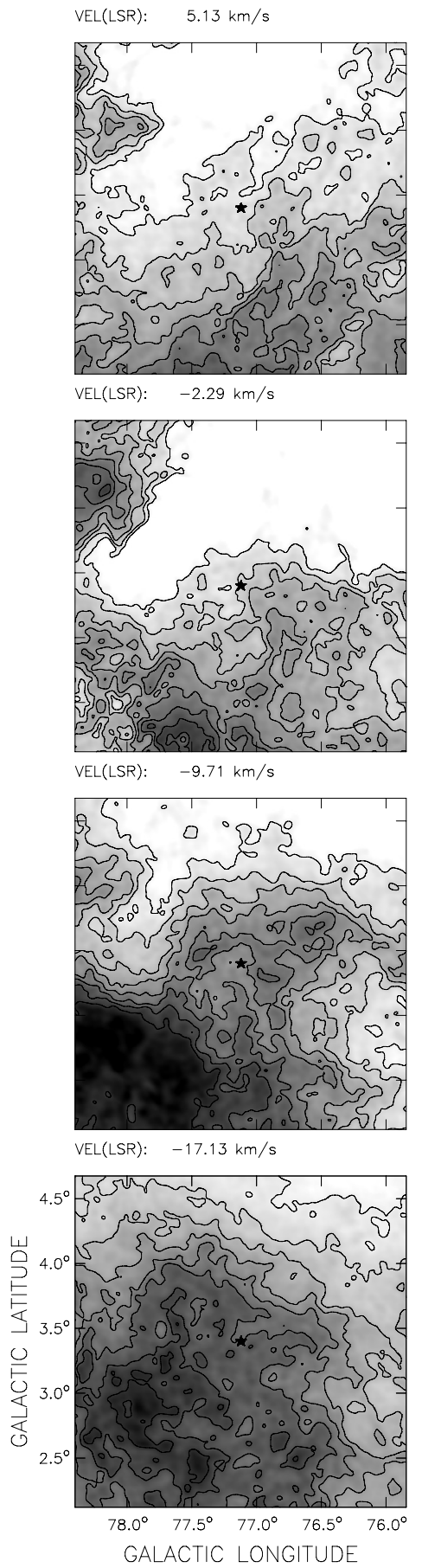
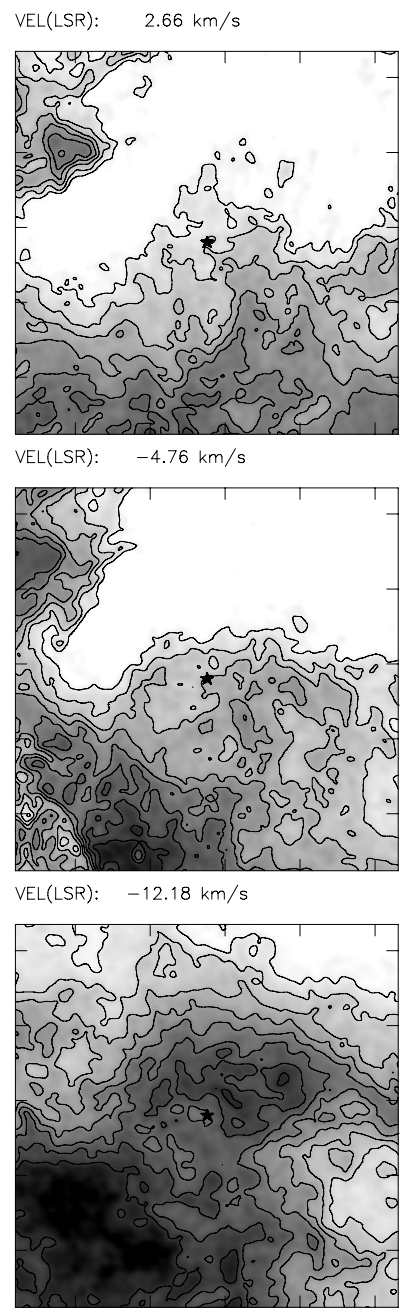

$\operatorname{VEL}(L S R): \quad-19.60 \mathrm{~km} / \mathrm{s}$

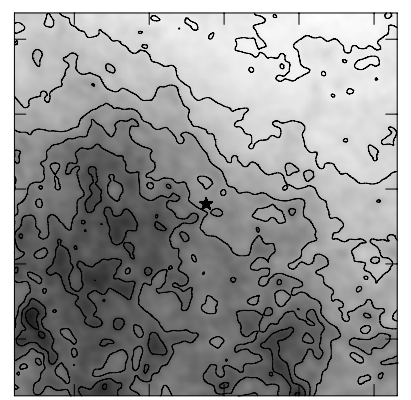

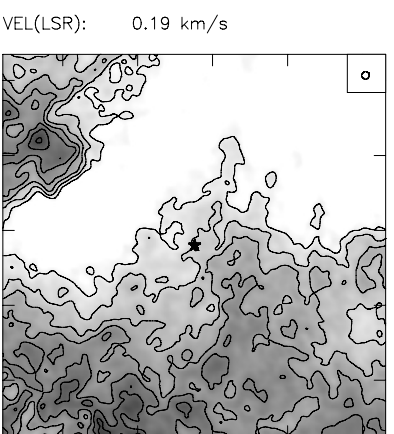

$\operatorname{VEL}(\operatorname{LSR}): \quad-7.23 \mathrm{~km} / \mathrm{s}$
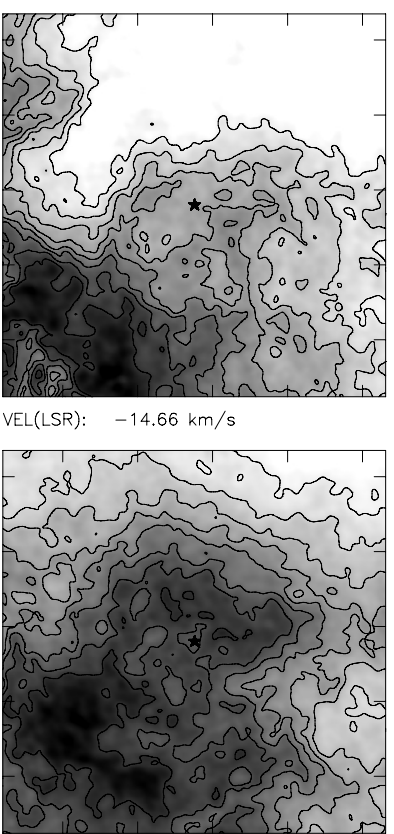

VEL(LSR): $\quad-22.08 \mathrm{~km} / \mathrm{s}$

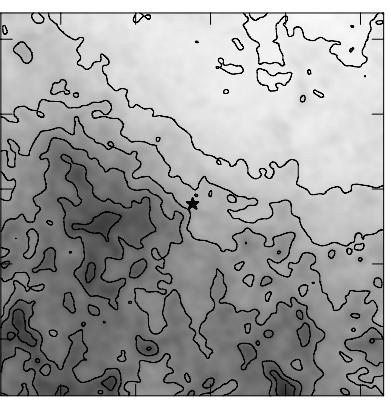

Fig. 5. H i brightness temperature images for selected velocity ranges. The velocity range covered by each map is $2.47 \mathrm{~km} \mathrm{~s}^{-1}$, and the displayed temperatures are a mean over the entire velocity map velocity width. The central velocity of each image is given in the upper left corner. The beam size is shown as a circle in the upper right corner of the entire figure. A constant mean brightness temperature value was subtracted from each image (see text). The lowest temperature contour is $-21 \mathrm{~K}$ and the contour spacing is $7 \mathrm{~K}$.

assuming that the fluxes at 60 and $100 \mu \mathrm{m}$ are accurate to within $21 \%$ and $32 \%$, respectively (Fich \& Terebey 1996). The derived dust temperature is typical of $\mathrm{H}$ II regions (e.g. Fich \& Terebey 1996).

The IR luminosity in the $7-135 \mu \mathrm{m}$ band can be calculated using the equations (Tokunaga 2000)

$L_{\mathrm{IR}}=3.112 \times 10^{7} \times d^{2} \times F_{\mathrm{IR}}\left(L_{\odot}\right)$

where $d$ is the distance in pc and $F_{\text {IR }}$ is the measured flux density given by

$$
F_{\mathrm{IR}}=a \times\left(b F_{12}+c F_{25}+d F_{60}+e F_{100}\right)\left(\mathrm{W} \mathrm{m}^{-2}\right)
$$

where $F_{i}$ is the measured flux density in band $i$ and $a, b, c, d$, and $e$ are constants whose values are $1.0 \times 10^{-14}, 20.653,7.538$, 4.578 and 1.762 , respectively. Based only on the flux density errors quoted in Table 3, which only reflect measurement errors, the $F_{\text {IR }}$ determination is accurate to within $6 \%$. Under the assumption that the HIRES fluxes are correctly calibrated, the $L_{\mathrm{IR}}$ estimate, $L_{\mathrm{IR}} \sim 8.8 \times 10^{4} L_{\odot}$ is accurate to within $\sim 40 \%$. The dust mass can be estimated from the measured $100 \mu \mathrm{m}$ flux, using

$M_{\mathrm{d}}=4.81 \times 10^{-12} \times F_{100} \times d^{2} \times\left[\left(\mathrm{e}^{\left(143.88 / T_{d}\right.}-1\right)\right]\left(M_{\odot}\right)$

where $F_{100}$ is the flux density at $100 \mu \mathrm{m}$ in Jy, $d$ the distance in $\mathrm{pc}$, and $T_{\mathrm{d}}$ the dust temperature in $\mathrm{K}$. A mass absorption 

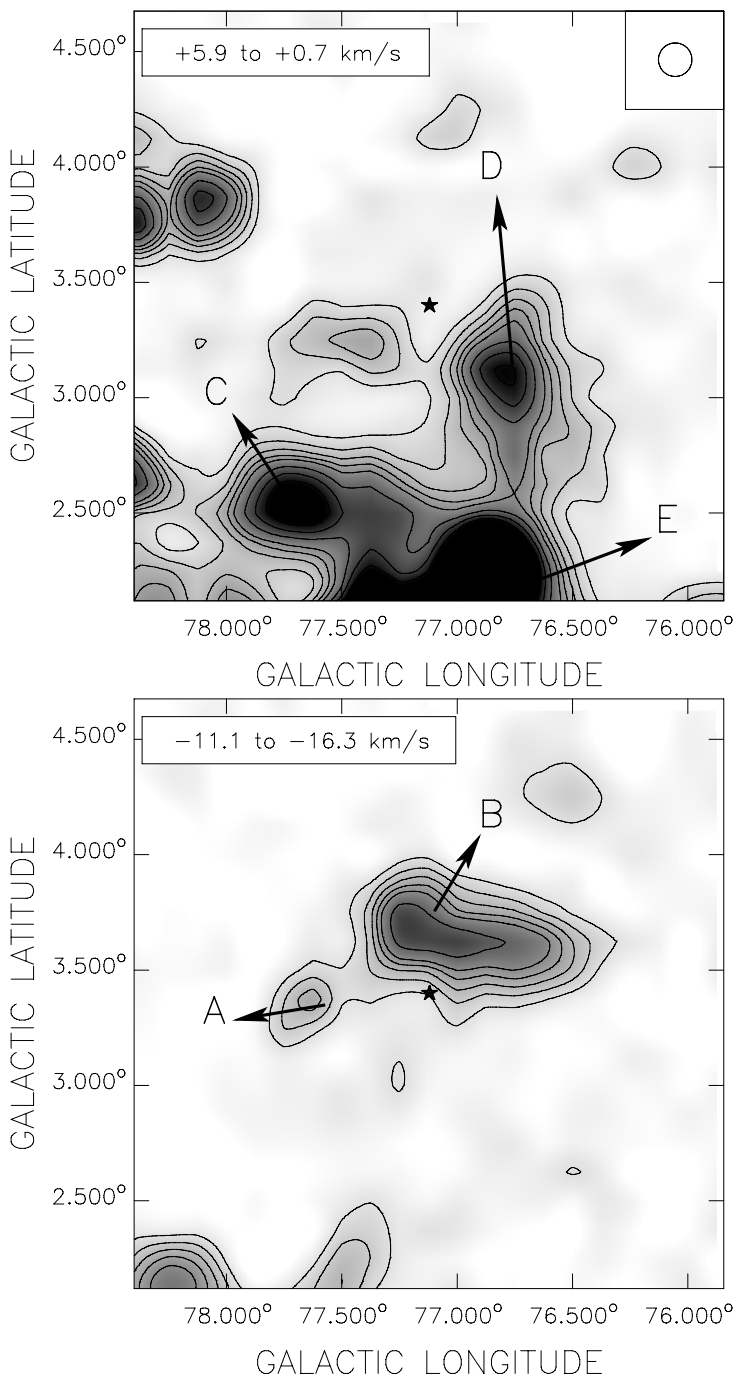

Fig. 6. $\mathrm{CO}$ emission distribution in the velocity range from +5.9 to $+0.7 \mathrm{~km} \mathrm{~s}^{-1}$ (upper panel) and from -11.1 to $-16.3 \mathrm{~km} \mathrm{~s}^{-1}$ (lower panel). Contour levels correspond to $0.7,1.2,1.7,2.2,2.7,3.7,4.7$, 5.7, and $6.7 \mathrm{~K}$. The location of HD 192281 is indicated by a star symbol. The main CO concentrations are labelled A, B, C, D, and E. The angular resolution is indicated in the top right corner of the upper panel.

coefficient per unit length of $2.5 \mathrm{~m}^{2} \mathrm{~kg}^{-1}$ at $100 \mu \mathrm{m}$ is assumed. The dust mass turns out to be $130 \pm 35 M_{\odot}$. The dust mass uncertainty is derived based on the uncertainties quoted for $F_{100}$, $T_{\mathrm{d}}$, and $d$.

\subsection{Carbon monoxide data}

The main physical characteristics of the different $\mathrm{CO}$ concentrations are summarized in Table 4.

The positions quoted for the concentrations correspond to the $\mathrm{CO}$ peak, and the LSR velocity, $V_{\mathrm{LSR}}$, is the velocity corresponding to the maximum $\mathrm{CO}$ temperature of that concentration. The line width at half maximum, $\Delta V$, was derived by fitting a Gaussian to the observed averaged profile. The linear dimensions were derived from a two-dimensional Gaussian fitting. The position angle (PA) of the concentration's major axis is measured counterclockwise from north. To estimate the mass, we used the empirical relationship between molecular hydrogen column density, $N\left(\mathrm{H}_{2}\right)$, and integrated $\mathrm{CO}$ intensity, $W\left({ }^{12} \mathrm{CO}\right)$,

$N\left(\mathrm{H}_{2}\right)=2.0 \times 10^{20} W\left({ }^{12} \mathrm{CO}\right)\left(\mathrm{cm}^{-2}\right)$,

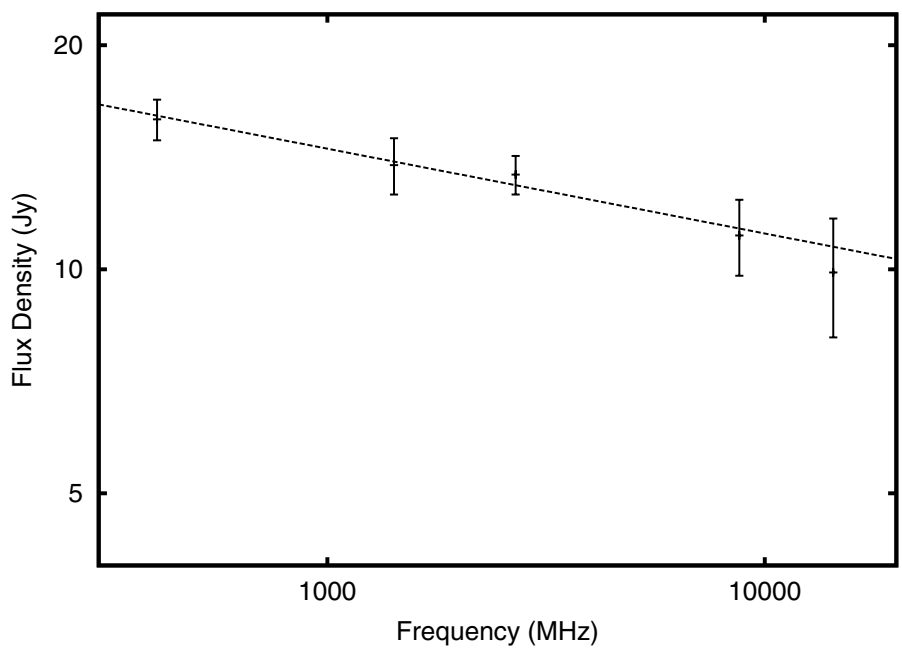

Fig. 7. Radio spectrum of the shell like feature observed in the neighborhood of HD 192281. Three $\sigma$ error bars are depicted along the measured flux density. The dashed line represents the flux density for an optically thin plasma having a flux density spectral index of $\alpha=-0.11$.

Table 3. Mid and far-infrared flux density measurements.

\begin{tabular}{ccc}
\hline \hline$\lambda(\mu \mathrm{m})$ & Database & Flux density $(\mathrm{Jy})$ \\
\hline 8.28 & MSX-A & $750 \pm 65$ \\
12.00 & HIRES & $1295 \pm 12$ \\
12.13 & MSX-C & $1035 \pm 60$ \\
14.65 & MSX-D & $370 \pm 70$ \\
21.30 & MSX-E & $737 \pm 110$ \\
25.00 & HIRES & $1035 \pm 130$ \\
60.00 & HIRES & $7555 \pm 545$ \\
100.00 & HIRES & $31720 \pm 1550$ \\
\hline
\end{tabular}

where $W\left({ }^{12} \mathrm{CO}\right)$ is given in units of $\mathrm{K} \mathrm{km} \mathrm{s}^{-1}$ (Magnani \& Onello 1995). The total mass of each concentration was estimated by using

$M=\mu m_{\mathrm{H}} d^{2} \sum N\left(\mathrm{H}_{2}\right) \Omega$,

where $d$ is the distance of the concentration, $\Omega$ is the solid angle subtended by the concentration, $m_{\mathrm{H}}$ is the proton mass, and $\mu$ is the mean molecular weight per $\mathrm{H}_{2}$ molecule. Assuming a relative helium abundance per mass of $25 \%, \mu$ turns out to be $\mu=2.8$. A volume density, $n\left(\mathrm{H}_{2}\right)$, was derived under the assumption that the total mass $M$ is distributed in a spherical volume of radius $R=\sqrt{L l}$, where $L$ and $l$ are the major and minor axes, respectively, of the observed angular distribution. These quantities were corrected for beam broadening.

\subsection{Neutral hydrogen data}

Taking the structures described in Sect. 3.3 into account, mean brightness temperature images spanning the velocity range from +3.5 to $-4.8 \mathrm{~km} \mathrm{~s}^{-1}$ and from -5.6 to $-14.7 \mathrm{~km} \mathrm{~s}^{-1}$ were constructed (Fig. 8). HD 192281, whose position is marked in both panels, is seen projected onto the outer part of the northern extreme of a semi-spherical feature (Fig. 8, left panel), and appears projected onto the interior of an $\mathrm{H}_{\mathrm{I}}$ elliptic feature (Fig. 8, right panel).

In the velocity range from -5.6 to $-14.7 \mathrm{~km} \mathrm{~s}^{-1}$, both the spatial H I distribution and the position of HD 192281 fullfill the first two criteria put forward in Sect. 3.3. On the other hand, strictly speaking, the negative value of the radial velocity of the 

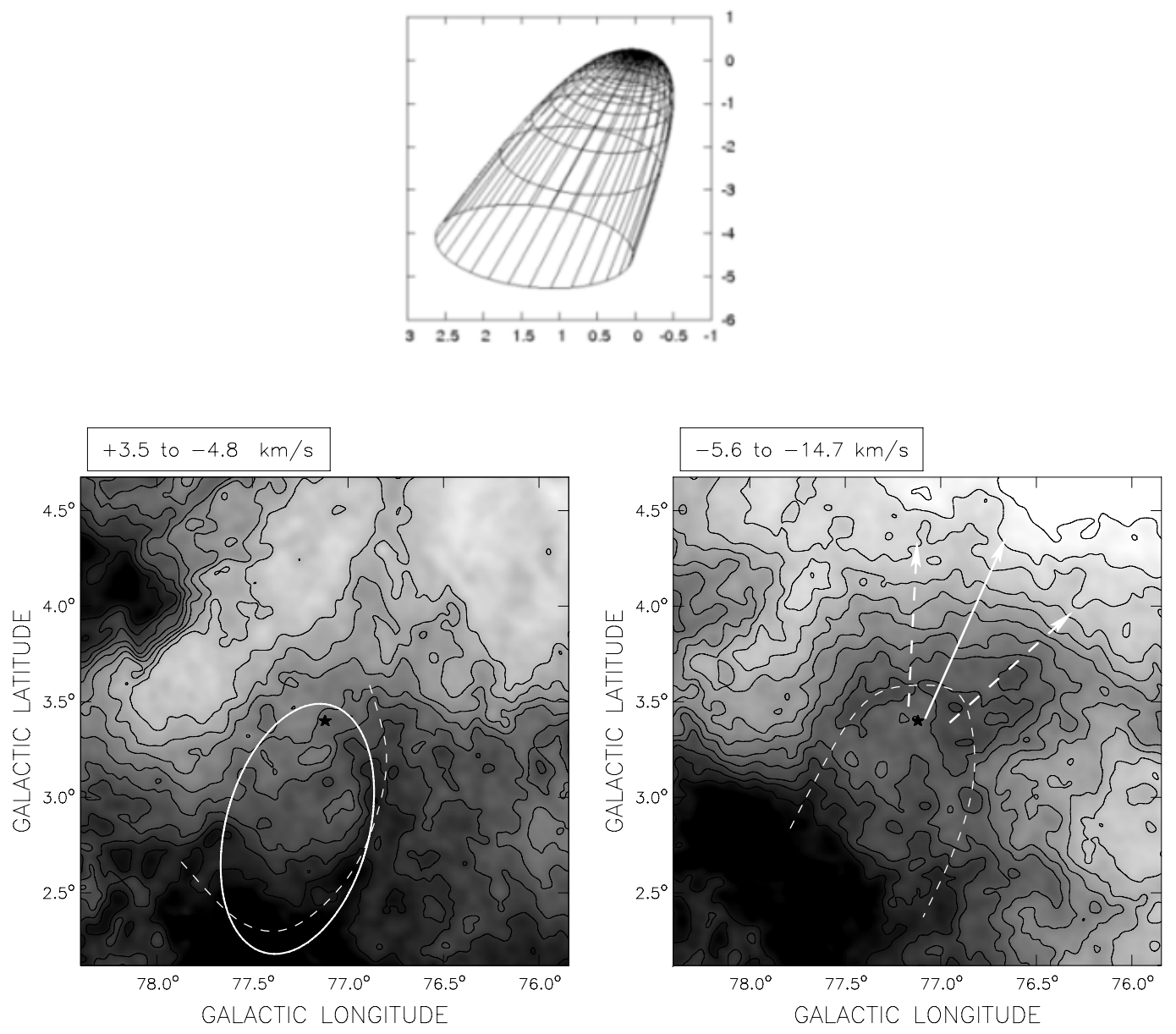

Fig. 8. H I temperature brightness emission distribution from +3.5 to $-4.8 \mathrm{~km} \mathrm{~s}^{-1}$ (lower left panel) and from -5.6 to $-14.7 \mathrm{~km} \mathrm{~s}$ (lower right panel). The stellar position is marked by a star symbol. A constant mean brightness temperature value was subtracted from each image (see text). The direction of the nominal peculiar stellar proper motion is shown by a solid arrow, while $\pm 1 \sigma$ directions are shown by dashed arrows (lower right panel). In both lower panels the outer border of the bow shock is indicated by a dashed curve. In the lower left panel the ellipse depicts the bow shock cross section at a distance of 1 pe downstream from the stellar position. The upper panel shows the 3D bow shock structure orientation as it would appear to an external observer (see text). The star is located at the $(0,0)$ position.

Table 4. Main physical parameters of the CO structures

\begin{tabular}{cccccccccc}
\hline \hline Feature & $l$ & $b$ & $\begin{array}{c}V_{\text {lsr }} \\
\left(\mathrm{km} \mathrm{s}^{-1}\right)\end{array}$ & $\begin{array}{c}L \\
(\mathrm{pc})\end{array}$ & $\begin{array}{c}l \\
(\mathrm{pc})\end{array}$ & $\begin{array}{c}\text { PA } \\
\left({ }^{\circ}\right)\end{array}$ & $\begin{array}{c}N\left(\mathrm{H}_{2}\right) \\
\left(10^{20} \mathrm{~cm}^{-2}\right)\end{array}$ & $\begin{array}{c}\text { Mass } \\
\left(M_{\odot}\right)\end{array}$ & $\begin{array}{c}n \\
\left(\mathrm{~cm}^{-3}\right)\end{array}$ \\
\hline $\mathrm{A}$ & 77.63 & +3.37 & -15.0 & 5.7 & 3.0 & 133 & 2.3 & 350 & 60 \\
$\mathrm{~B}$ & 77.15 & +3.63 & -13.4 & 20.0 & 5.7 & 85 & 33.7 & 37800 & 210 \\
$\mathrm{C}$ & 77.67 & +2.52 & +2.3 & 7.0 & 7.0 & - & 34.7 & 8900 & 210 \\
$\mathrm{D}$ & 76.76 & $+3^{\circ} .11$ & +3.0 & 20.0 & 6.3 & 8 & 9.1 & 5550 & 90 \\
$\mathrm{E}$ & 76.87 & +2.25 & +1.3 & 12.2 & 6.4 & 90 & 9.1 & 3700 & 60 \\
\hline
\end{tabular}

H I minimum rules out any possible physical relationship between the $\mathrm{H}$ I gas and the star because the distance corresponding to an $\mathrm{H}_{\mathrm{I}}$ structure having a radial velocity of $-10 \mathrm{~km} \mathrm{~s}^{-1}$ would be $5.6 \pm 1.0 \mathrm{kpc}$, while distance estimates for HD 192281 vary between 1.55 and $2.4 \mathrm{kpc}$. However, as mentioned before, in this part of the Galaxy a straightforward interpretation of kinematic distances should be treated with caution. Along the line of sight towards HD 192281 kinematic distances are unreliable due to both the small velocity gradient predicted by galactic rotation models and the presence of streaming motions (Burton 1974).

In this context, it is worth mentioning that Hron (1987) lists four open clusters (see his Table 1a) in the galactic longitude range $75^{\circ} \leq l \leq 79^{\circ}$, which have mean radial velocities in the range from -13 to $+4 \mathrm{~km} \mathrm{~s}^{-1}$, even though their photometric distances fall between 1.02 and $1.86 \mathrm{kpc}$. He claims that these clusters have radial velocities and distances accurate to $4 \mathrm{~km} \mathrm{~s}^{-1}$ and $20 \%$, respectively. The galactic velocity field of Fich et al. (1989) would place these clusters at kinematic distances ranging from $3.9 \mathrm{kpc}$ to $6.0 \mathrm{kpc}$. Thus, it would be safe to conclude that along $l \sim 77^{\circ}$ a negative radial velocity does not necessarily imply a long distance from the Sun.

Bearing in mind that an early O-type star is expected to stir up its surrounding ISM, and since throughout the entire $\mathrm{H}_{\mathrm{I}}$ data cube we could not find another region of low neutral hydrogen emissivity close enough to HD 192281, we believe that the $\mathrm{H}$ I structures shown in Fig. 8 are the result of the interaction of HD 192281 with its surroundings. 


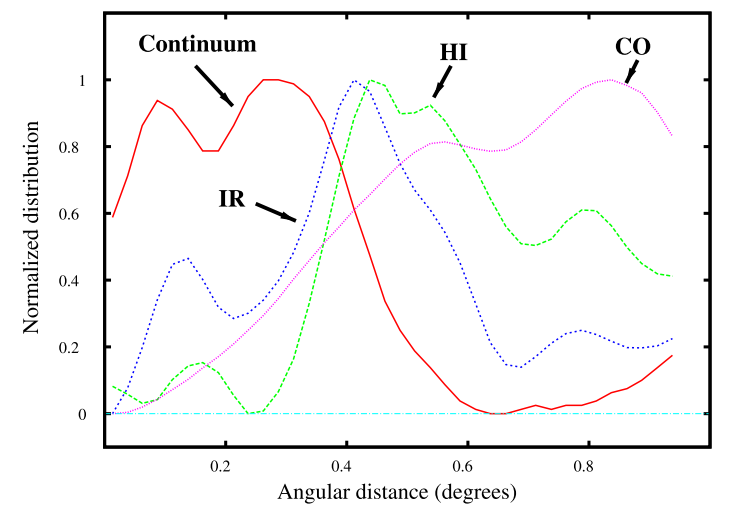

Fig. 9. Radial distribution of the continuum emission at $2695 \mathrm{MHz}$ (red line), $60 \mu \mathrm{m}$ (blue line), $\mathrm{H}$ i (green line), and $\mathrm{CO}$ emission (magenta line) as a function of the angular distance from $(l, b)=\left(77^{\circ} .3,+3^{\circ} .0\right)$.

\subsection{Relationship among different structures}

Morphological evidence in favour of a physical association between the ridge of $\mathrm{CO}$ (see Fig. 6, upper panel), the H I emission (see Fig. 8, left panel), the continuum emission, and the IR can be seen from a comparison of their distribution as a function of angular distance (Fig. 9) from the geometric centre, $(l$, $b)=\left(77^{\circ} .3,+3^{\circ} .0\right)$ of the H I feature. In Fig. 9 the different lines represent the mean value of the corresponding emission along concentric rings spaced by 1'.5. The average was taken between position angles, measured counterclockwise from north, 207.0 and 298.0. The continuum, infrared, and $\mathrm{H}_{\mathrm{I}}$ images were first convolved to a common beam of 4.3. The normalized emission at a given frequency was computed by subtracting from each point the minimum value at that frequency $[0.32 \mathrm{~K}(2695 \mathrm{MHz})$, $7.14 \mathrm{~K}(\mathrm{HI}), 85.8 \mathrm{MJy} / \mathrm{sr}(60 \mu \mathrm{m})$, and $0.03 \mathrm{~K}(\mathrm{CO})]$, and then dividing by the corresponding maximum $[0.8 \mathrm{~K}(2695 \mathrm{MHz})$, $12.3 \mathrm{~K}\left(\mathrm{HI}_{\mathrm{I}}\right), 101 \mathrm{MJy} / \mathrm{sr}(60 \mu \mathrm{m})$ and $\left.0.95 \mathrm{~K}(\mathrm{CO})\right]$. This comparison shows that the ionized gas depicts the innermost distribution, where the molecular gas is the outermost one with the infrared and atomic gas peaking between them. These results are consistent with what would be expected for an arc-like feature that is being heated, ionized, and dissociated from a central source.

In Fig. 6 (lower panel) it is shown that HD 192281 is seen projected onto a small indentation of the southern border of the $\mathrm{CO}$ feature labelled $\mathrm{B}$. At first sight, the physical location and the mean radial velocity $\left(-13.4 \mathrm{~km} \mathrm{~s}^{-1}\right)$ of the molecular concentration may speak in favour of a physical association between this molecular feature and the ovoid $\mathrm{H}_{\mathrm{I}}$ feature whose systemic velocity is $\sim-10 \mathrm{~km} \mathrm{~s}^{-1}$. If the observed angular distance between concentration B and HD 192281 were a consequence of a short physical distance between them, the molecular gas would be exposed to a very high flux of stellar ionizing photons. Therefore, the rain of energetic photons from HD 192281 would inevitably provoke dissociation of the molecular gas, ionization of the atomic gas, and heating of the dust particles. Owing to this, we would expect, in the immediate neighbourhood of HD 192281, to observe radio continuum emission (arising from the ionized gas), infrared emission (arising from the heated dust), and emission arising from polycyclic aromatic hydrocarbons (PAH). The latter absorb UV radiation and re-emit in various mid-infrared spectral features. The $8.3 \mu \mathrm{m}$ MSX Band contains two of the strongest PAH features at 7.7 and $8.6 \mu \mathrm{m}$. For this reason, this band is an excellent tracer of a photodissociation region (PDR), which is the interface between atomic

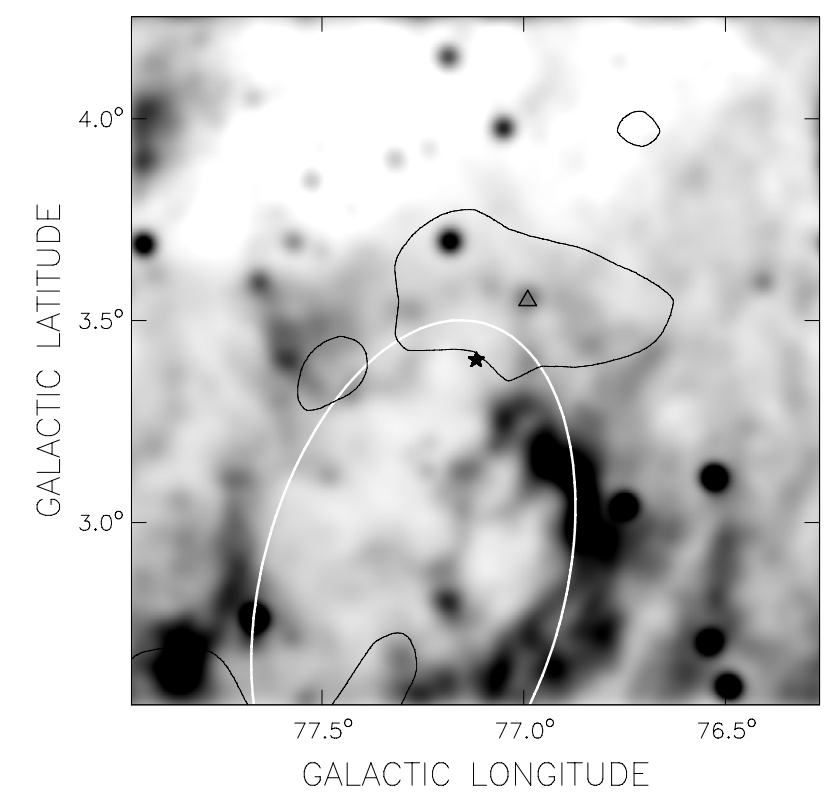

Fig. 10. MSX Band A ( $8.3 \mu \mathrm{m}$, gray scale $)$ emission distribution convolved to a 3 arc min resolution. The solid contour indicates the location of the CO clouds labelled A and B (see Fig. 6). The star indicates the position of HD 192281, and the triangle marks the position of the YSO candidate IRAS 20097+4005. The ellipse depicts the cross section of the bow shock structure corresponding to $z=1 \mathrm{pc}$ downstream the apex of the bow shock.

and molecular gas. Though a close examination of the databases used in this work shows no increment in the expected continuum emission in both the radio and the IR windows in the region of structure B facing the position of HD 192281, there seems to be a small feature close to the star in the MSX Band A that may be pinning down the location of a PDR in this molecular concentration.

In Fig. 10, projected onto the area covered by cloud B, we can see a weak arc of $8.3 \mu \mathrm{m}$ emission and the IRAS point source IRAS20097+4005. This weak arc of emission is not seen at the other MSX bands. Based on this, we identify the $8.3 \mu \mathrm{m}$ emission with a PDR created by HD 192281 within cloud B. Applying the criteria of Junkes et al. (1992, JFR) we find that IRAS20097+4005, which is seen projected onto the PDR, fullfills the requirements set by JFR to be considered a young stellar object (YSO) candidate. The observed morphology suggests that the expansion of the ionized region could have induced the formation of a new star in the neighbouring molecular envelope.

With the information at hand, and bearing in mind the lack of both radio continuum and IR emission already reported in IBs associated with other early type stars (Cichowolski \& Arnal 2004), a physical association between the $\mathrm{H}_{\mathrm{I}}$ feature related to HD 192281 and concentration B cannot be excluded.

\subsection{A bow shock scenario}

The different databases used in this investigation are consistent with a scenario where HD 192281 has strongly influenced its surrounding interstellar medium. In particular, the $\mathrm{H}_{\mathrm{I}}$ data show two structures at slightly different radial velocities. One of the structures has the appearance of a semi-circular feature (Fig. 8, left panel) while the other looks like an ellipsoid (Fig. 8, right panel). In this context, we wonder whether these findings could be supported by a bow shock model. The first relevant issue is whether HD 192281 can be considered a runaway star. By 
adopting a radial velocity of $-23.1 \pm 8 \mathrm{~km} \mathrm{~s}^{-1}$ (De Becker \& Rauw 2004) and following the procedure outlined by van der Sluys \& Lamers (2003) (after correcting the sign of the $w_{\odot}$ term in their Eq. (19)) and Cichowolski et al. (2008), and by adopting a distance of $1.5 \pm 0.3 \mathrm{kpc}$ for HD 192281, the stellar peculiar velocity components are

$$
\begin{aligned}
& v_{\text {pec }, r}=-15.3 \pm 8.8 \mathrm{~km} \mathrm{~s}^{-1} \\
& v_{\text {pec }, l}=-11.2 \pm 13.1 \mathrm{~km} \mathrm{~s}^{-1} \\
& v_{\text {pec }, b}=34.0 \pm 10.0 \mathrm{~km} \mathrm{~s}^{-1}
\end{aligned}
$$

where $v_{\text {pec }, r}$, is the peculiar radial velocity, $v_{\text {pec }, l}$ the peculiar velocity along galactic longitude, and $v_{\mathrm{pec}, b}$ the peculiar velocity along galactic latitude. The corresponding total peculiar velocity of the star,

$v_{\mathrm{pec}}=\sqrt{v_{\mathrm{pec}, r}^{2}+v_{\mathrm{pec}, l}^{2}+v_{\mathrm{pec}, b}^{2}}$

is $v_{\text {pec }}=39.0 \pm 10.0 \mathrm{~km} \mathrm{~s}^{-1}$. Increasing the stellar distance to its maximum quoted value, namely $2.4 \mathrm{kpc}$, the total peculiar velocity increases to $v_{\text {pec }}=55.0 \pm 15.0 \mathrm{~km} \mathrm{~s}^{-1}$. Therefore, when adopting the criterion of Cruz-González et al. (1974), the spatial peculiar velocity of this star (either at $1.5 \mathrm{kpc}$ or $2.4 \mathrm{kpc}$ ) implies that HD 192281 is very likely a runaway star.

According to van der Sluys \& Lamers (2003) only two angles, labelled $i$ and $\phi$, are needed to completely define the spatial orientation with respect to the observer of the structure created by a star moving with respect to its ISM with a high peculiar velocity. The angle $i$ is measured from the line of sight towards the observer ( $i=90^{\circ}$ corresponds to the plane of the sky), whilst $\phi$ is measured on the plane of the sky, counterclockwise from the tip of the peculiar velocity vector (see Fig. 4 of van der Sluys \& Lamers 2003). Following van der Sluys \& Lamers (2003), the above angles are defined as

$$
\begin{gathered}
i=\arcsin \left(\frac{v_{\mathrm{pec}, r}}{v_{\mathrm{pec}}}\right) \\
\phi=\arctan \left(\frac{v_{\mathrm{pec}, l}}{v_{\mathrm{pec}, b}}\right) .
\end{gathered}
$$

After adopting a stellar distance of $1.5 \pm 0.3 \mathrm{kpc}$, the angles are $i=113^{\circ} \pm 11^{\circ}$, and $\phi=-18^{\circ} \pm 20^{\circ}$, while at the largest distance quoted for HD 192281, $2.4 \mathrm{kpc}$ (Humphreys 1978), the angles $i$ and $\phi$ are $105^{\circ} \pm 15^{\circ}$, and $-18^{\circ} \pm 21^{\circ}$, respectively. Therefore, an increment in the distance has little effect on the overall orientation of the structure. The angles $i$ and $\phi$ remain, within their errors, almost unchanged.

The shape of the structure formed by a fast-moving star has been the subject of several papers (Wilkin 1996; Raga et al. 1997). All of them indicate that such a star should create a bow shock structure whose symmetry axis should be aligned with the direction of motion of the star. To model this structure, we follow Wilkin (1996) by assuming a homogeneous interstellar medium with density $\rho$, and an isotropic stellar wind with constant mass-loss rate $\dot{M}$ and terminal velocity $v_{\infty}$. The resulting structure should have a paraboloidal shape, with the stellar velocity vector as its symmetry axis, and a stand-off distance $r_{\mathrm{O}}$ (i.e. the distance from the star along the symmetry axis where the momentum of the stellar wind and the ambient medium balance each other) given by

$r_{\mathrm{o}}=1.77 \times 10^{3} \times \sqrt{\frac{\dot{M} v_{\infty}}{\mu n_{\mathrm{o}} v_{\mathrm{pec}}^{2}}}(\mathrm{pc})$, where $\dot{M}$ is given in units of $M_{\odot} / \mathrm{yr}, v_{\infty}$ and $v_{\text {pec }}$ in $\mathrm{km} \mathrm{s}^{-1}, n_{\mathrm{o}}$ in units of $\mathrm{cm}^{-3}$, and $\mu$ is the mean molecular weight. Since HD 192281 has no measured values of $\dot{M}$ and $v_{\infty}$, we have adopted for this star those provided for HD 14434, a star with a similar spectral type (Chlebowski \& Garmany 1991). Hence, $\dot{M}=7 \times 10^{-7} M_{\odot} / \mathrm{yr}$, and $v_{\infty}=2700 \mathrm{~km} \mathrm{~s}^{-1}$. Adopting $\mu=1.3$ for the $\mathrm{H}_{\mathrm{I}}$ we obtain $1.4 n_{\mathrm{o}}^{-0.5} \leq r_{\mathrm{o}} \leq 2.1 n_{\mathrm{o}}^{-0.5}$. The lowest value of $r_{\mathrm{o}}$ corresponds to the largest value of $v_{\mathrm{pec}}$ (e.g. the highest distance for HD 192281). After error propagation, the relative error of $r_{\mathrm{o}}$ is of the order of $27 \%$.

Due to projection effects, the stand-off distance given by Eq. (9) is related to the observed stand-off distance $\left(r_{\mathrm{o}, \mathrm{p}}\right)$ by

$r_{\mathrm{o}, \mathrm{p}}=r_{\mathrm{o}} \sin (i) \cos (\phi)$,

where the angles $i$ and $\phi$ are those defined above. Adopting a stellar distance of $1.5 \mathrm{kpc}$, the projected angular distance between the star and the apex of the bow-shock would be $r_{\mathrm{o}, \mathrm{p}}=$ $(3.5 \pm 0.7) n_{\mathrm{o}}^{-0.5}$. The quoted error stems from the errors in both angles. From Fig. 8 (right panel), a $\mathrm{H}_{\mathrm{I}}$ profile showing the variation with distance from HD 192281 of the H I mean brightness temperature was constructed by integrating the $\mathrm{H}_{\mathrm{I}}$ emission along concentric rings spaced by $72^{\prime \prime}$. From this profile (not shown here) we derived an angular offset of $\left(5{ }^{\prime} 4 \pm 1 ! 2\right)$ between the star and the peak of the Hi emission. This peak approximately pinpoints the position on the plane of the sky of the apex of the $\mathrm{H}_{\mathrm{I}}$ bow-shock structure, implying $n_{\mathrm{o}} \sim 0.4 \pm 0.6 \mathrm{~cm}^{-3}$. This value of $n_{\mathrm{o}}$ is, within its large uncertainty, in reasonable agreement with the number density expected, $n_{\mathrm{o}} \sim 0.2 \mathrm{~cm}^{-3}$ (Lockman 1984), at the $z$ distance of HD $192281(z \sim 90 \mathrm{pc})$ from the galactic plane.

In the right panel of Fig. 8, it can be seen that the tangential peculiar motion of the star is oriented towards increasing latitude and decreasing longitude. From the radial and tangential peculiar velocities, the orientation of the bow shock structure with respect to the observed $\mathrm{H}_{\mathrm{I}}$ brightness temperature distribution is shown by the upper panel in Fig. 8 .

It can be reasonably argued that the observed $\mathrm{H}_{\mathrm{I}}$ distribution shown in Fig. 8 (right panel) is consistent with a bow shock geometry. Furthermore, part of the arc-like feature seen in the $\mathrm{H}$ I distribution at more positive velocities (see Fig. 8, left panel) may be interpreted as belonging to the surface defining the bow shock downstream of the apex.

According to Wilkin (1996) the velocity of the gas moving downstream from the apex along the surface of the bow shock, also denominated tangential velocity, reaches, for low values of the ratio $v_{\text {pec }} / v_{\infty}$ and large $\theta$ angles from the axis of symmetry, a value that is close to the stellar peculiar spatial velocity. The angle $\theta$ is a polar angle measured from the axis of symmetry of the bow shock, as seen by the star at the coordinate origin (the direction along $\theta=0^{\circ}$ points towards the head of the bow shock). In the case of HD 192281 this implies that the tangential velocity may reach a value as high as $\sim 39 \mathrm{~km} \mathrm{~s}^{-1}$ (assuming the star is located at $1.5 \mathrm{kpc}$ ). Projection effects mean that the maximum radial velocity spread of any neutral gas associated with the bow shock would be $\sim 34 \mathrm{~km} \mathrm{~s}^{-1}$. If our interpretation were correct, bearing in mind that HD 192281 is approaching the observer, the atomic gas on the surface of the bow shock would be detectable at radial velocities that are more positive than the radial velocity of the H I gas observed close to the apex. This is in good agreement with the observations. In this context, the large velocity interval covered by the H I gas likely to be related to the bow shock, from +3.5 to $-14.7 \mathrm{~km} \mathrm{~s}^{-1}$, is easily accounted for.

Though the global morphology predicted by the bow shock model shows reasonable agreement with observational findings, 
there are differences between model predictions and the observations that cannot be explained. Indeed, under the assumption of a continuous mass loss from the star and a homogeneous ISM, we would expect to have a higher gas density close to the apex. Since this material with higher density should be located closer to the star than the material belonging to other parts of the bow shock, we would expect to have some relative bright radio continuum and infrared emissions outlining the interior of the bow shock in the apex and somewhat near the apex. Our observations do not show such features. In spite of these drawbacks, we are fairly confident that most of our observational findings towards the ISM around HD 192281 are in good agreement with and are explained well by a bow shock model.

\section{Is HD 192281 associated with G78.2+2.1?}

It is well known that, when the primary component of a massive binary system explodes as a supernova, the secondary may start moving with a spatial velocity comparable to its original orbital velocity (30 to $150 \mathrm{~km} \mathrm{~s}^{-1}$ ). This is one of the scenarios, the so-called binary-supernova scenario (or BSS for short), put forward to explain the origin of runaway stars (Burrows et al. 1995). HD 192281 has a spatial velocity compatible with a mild runaway nature, and the path of its proper motion when extrapolated backward in time passes close to the centre of the SNR G78.2+2.1. Since both objects appear to have comparable distances $(\sim 1.5 \mathrm{kpc})$, it may be worth exploring the possibility further that the origin of the high spatial velocity of HD 192281 is consequence of a BSS. The angular distance between the star and the $S N R$ is $\sim 1.7$. The $S N R$ age varies between 4000 to $7000 \mathrm{yr}$ (Mavromatakis 2003). Therefore, to reach its present position with respect to the centre of G78.2+2.1, HD 192281 should have a proper motion between $\sim 0$.' $^{\prime}$ and $\sim 1^{\prime \prime} .5$ per year. These figures are two orders of magnitude larger than the measured stellar proper motion. Therefore, it is highly unlikely that both objects are genetically linked to each other.

\section{Conclusions}

Based on CGPS radio continuum and H I line data, MSX and IRAS high-resolution infrared data, radio continuum data at higher frequencies, and low-resolution CO data, the interstellar medium in the environs of the massive star HD 192281 was analysed. The main findings of our study can be summarized as follows:

a) The neutral hydrogen data show the presence of an elongated structure likely to be associated with HD 192281. The massive star is off-centred and located close to the high galactic latitude extreme of the $\mathrm{H}_{\mathrm{I}}$ feature. The atomic gas covers the velocity range from $\sim+4$ to $\sim-15 \mathrm{~km} \mathrm{~s}^{-1}$.

b) The H I feature has counterparts at both IR and radio continuum frequencies, and at molecular gas $(\mathrm{CO})$ emission.

c) The radio continuum emission is thermal (flux spectral index $\alpha=-0.11 \pm 0.02$ ), and HD 192281 is the likely source of ionization. The total mass of ionized gas is $1300 \pm 650 M_{\odot}$, and the mean electron density is $7.3 \pm 0.8 \mathrm{~cm}^{-3}$.

d) The IR feature almost mimics the radio continuum emission. A dust temperature of $24 \pm 4 \mathrm{~K}$ was derived using 60 and $100 \mu \mathrm{m}$ HIRES data. The total luminosity of this feature in the range from 7 to $135 \mu \mathrm{m}$ is $(8.8 \pm 3.5) 10^{4} L_{\odot}$. The total dust mass involved in the IR emission amounts to $130 \pm 35 M_{\odot}$. e) HD 192281 has a peculiar spatial velocity of $39.0 \pm$ $10.0 \mathrm{~km} \mathrm{~s}^{-1}$, and can be classified as a mild run-away star. In this context most of the observational findings are explained well by a bow shock model.

f) The interaction of the stellar wind of HD 192281 with the molecular cloud B may have induced star formation in the latter. In this context IRAS20097+4005 may be identified as a YSO candidate.

g) Based on the measured proper motion for HD 192281 and the accepted age for the SNR G78.2+2.1, there is no way of physically linking both objects.

Acknowledgements. This work was partially supported by the Agencia Nacional de Promoción Científica y Tecnológica (ANPCyT) under project PICT 14018/03 and 00812, by Consejo Nacional de Investigaciones Científicas y Técnicas CONICET under projects PIP 01299 and 02488, by Universidad Nacional de Buenos Aires (UBA) under project UBACyT X482, and by Universidad Nacional de La Plata (UNLP) under projects 11/G091 and 11/G093. The work of SP was supported by the Natural Sciences and Engineering Research Council of Canada (NSERC) and the Fonds FQRNT of Québec. The DRAO Synthesis Telescope is operated as a national facility by the National Research Council of Canada. The CGPS is a Canadian project with international partners and is supported by grants from NSERC. Data from the CGPS are publicly available through the facilities of the Canadian Astronomy Data Centre (http:// cadc.hia.nrc.ca) operated by the Herzberg Institute of Astrophysics, NRC. SP thanks the Instituto Argentino de Radioastronomía, where part of this work was carried, for its hospitality.

\section{References}

Avedisova, V. S. 1972, SvA, 15, 708

Barannikov, A. A. 1993, Astron. Lett., 19, 420

Burrows, A., Hayes, J., \& Fryxell, B. A. 1995, ApJ, 450, 830

Burton, W. B. 1974, in Galactic Radio Astronomy, ed. F. J. Kerr, \& S. C. Simonson, IAU Symp., 60, 551

Cao, Y., Terebey, S., Prince, T. A., \& Beichman, C. A. 1997, ApJS, 111, 387

Cappa, C. E., \& Herbstmeier, U. 2000, AJ, 120, 1963

Cappa, C., Pineault, S., Arnal, E. M., \& Cichowolski, S. 2002, A\&A, 395, 955

Cappa, C. E., Arnal, E. M., Cichowolski, S., Goss, W. M., \& Pineault, S. 2003, in A Massive Star Odyssey: From Main Sequence to Supernova, ed. K. van der Hucht, A. Herrero, \& C. Esteban, IAU Symp., 212, 596

Chaisson, E. J. 1976, in Front. Astrophys., 259

Chlebowski, T., \& Garmany, C. D. 1991, ApJ, 368, 241

Cichowolski, S., \& Arnal, E. M. 2004, A\&A, 414, 203

Cichowolski, S., Arnal, E. M., Cappa, C. E., Pineault, S., \& St-Louis, N. 2003, MNRAS, 343, 47

Cichowolski, S., Pineault, S., Arnal, E. M., \& Cappa, C. E. 2008, A\&A, 478, 443

Conti, P. S., \& Ebbets, D. 1977, ApJ, 213, 438

Cruz-González, C., Recillas-Cruz, E., Costero, R., Peimbert, M., \& TorresPeimbert, S. 1974, Rev. Mex. Astron. Astrofis., 1, 211

Dame, T. M., Ungerechts, H., Cohen, R. S., et al. 1987, ApJ, 322, 706

De Becker, M., \& Rauw, G. 2004, A\&A, 427, 995

Eenens, P. R. J., \& Williams, P. M. 1994, MNRAS, 269, 1082

Fich, M., \& Terebey, S. 1996, ApJ, 472, 624

Fich, M., Blitz, L., \& Stark, A. A. 1989, ApJ, 342, 272

Fowler, J. W., \& Aumann, H. H. 1994, in Science with High Spatial Resolution Far-Infrared Data, ed. S. Terebey, \& J. M. Mazzarella, 1

Fürst, E., Reich, W., Reich, P., \& Reif, K. 1990, A\&AS, 85, 691

Gies, D. R., \& Bolton, C. T. 1986, ApJS, 61, 419

Haslam, C. G. T. 1985, Bull. Inf. CDS, 28, 49

Haverkorn, M., Gaensler, B. M., McClure-Griffiths, N. M., Dickey, J. M., \& Green, A. J. 2006, ApJS, 167, 230

Higgs, L. A., \& Tapping, K. F. 2000, AJ, 120, 2471

Hog, E., Fabricius, C., Makarov, V. V., et al. 2000, VizieR Online Data Catalog, 1259

Hron, J. 1987, A\&A, 176, 34

Humphreys, R. M. 1978, ApJS, 38, 309

Junkes, N., Fürst, E., \& Reich, W. 1992, A\&A, 261, 289

Ladouceur, Y., \& Pineault, S. 2008, A\&A, 490, 197

Landecker, T. L., Dewdney, P. E., Burgess, T. A., et al. 2000, A\&AS, 145, 509

Langston, G., Minter, A., D'Addario, L., et al. 2000, AJ, 119, 2801

Lockman, F. J. 1984, ApJ, 283, 90

Lozinskaya, T. A. (ed.) 1992, Supernovae and stellar wind in the interstellar medium (New York: AIP) 
E. M. Arnal et al.: Structure and kinematics of ISM around HD 192281

Magnani, L., \& Onello, J. S. 1995, ApJ, 443, 169

Martins, F., Schaerer, D., \& Hillier, D. J. 2005, A\&A, 436, 1049

Mavromatakis, F. 2003, A\&A, 408, 237

McClure-Griffiths, N. M., Dickey, J. M., Gaensler, B. M., et al. 2005, ApJS, 158, 178

Mezger, P. G., \& Henderson, A. P. 1967, ApJ, 147, 471

Moffat, A. F. J., Shara, M. M., Smith, L. F., et al. 1998, JRASC, 92, 312

Pineault, S., Arnal, E. M., Cappa, C., et al. 2008, MNRAS, 386, 1739

Price, S. D., Egan, M. P., Carey, S. J., Mizuno, D. R., \& Kuchar, T. A. 2001, AJ, 121,2819

Quireza, C., Rood, R. T., Bania, T. M., Balser, D. S., \& Maciel, W. J. 2006, ApJ, 653,1226

Raga, A. C., Noriega-Crespo, A., Cantó, J., et al. 1997, Rev. Mex. Astron. Astrofis., 33, 73
Reich, P., Reich, W., \& Furst, E. 1997, A\&AS, 126, 413

Schraml, J., \& Mezger, P. G. 1969, ApJ, 156, 269

Schwartz, P. R. 1982, ApJ, 252, 589

Stil, J. M., Taylor, A. R., Dickey, J. M., et al. 2006, AJ, 132, 1158

Stone, R. C. 1978, AJ, 83, 393

Taylor, A. R., Gibson, S. J., Peracaula, M., et al. 2003, AJ, 125, 3145

Tokunaga, A. T. 2000, Infrared Astronomy (Allen's Astrophysical Quantities), 143

van der Sluys, M. V., \& Lamers, H. J. G. L. M. 2003, A\&A, 398, 181

Walborn, N. R. 1972, AJ, 77, 312

Weaver, R., McCray, R., Castor, J., Shapiro, P., \& Moore, R. 1977, ApJ, 218, 377

Wilkin, F. P. 1996, ApJ, 459, L31

Willis, A. G. 1999, A\&AS, 136, 603 